\title{
Interactive comment on "Atmospheric
} convergence zones stemming from large-scale mixing” by Gabriel M. P. Perez et al.

Gabriel M. P. Perez et al.

gabrielmpp@protonmail.com

Received and published: 25 March 2021

article 


\section{Reply to anonymous Reviewer 2}

Interactive

Gabriel M P Perez

comment

March 2021

\section{Response to general comments}

We appreciate and thank the Reviewer for the insightful comments and suggestions. The Reviewer pointed out the following general issues:

1 - Abbreviations We have addressed the issue of unexplained abbreviations in the revised manuscript.

2 - Title We have considered the Reviewer's suggestion about including "Coherent structures" in the title. We will be adding these as keywords for the final manuscript. However, our analysis is not limited to coherent structures (FTLE ridges). It also includes correlations between a metric of mixing efficiency (the FTLE scalar field) and water vapour and rainfall. Since "coherent structures" can be studied within the framework of chaotic mixing, we believe that the current title better express the contents of the paper. 


\section{Response to minor comments}

\subsubsection{Line 45}

We thank the Reviewer for this suggestion. We have detailed the definition of conver-

Interactive gence zone as suggested.

comment

2.0.2 Further approaches do not count elongated structures as coherent sets, why does this work here?

We thank the Reviewer for this comment. However, we are not entirely sure about which part of the manuscript the Reviewer is referring to here. We do describe the LCSs on our case study as a "coherent ensemble" because they last for a considerable amount of time and seem to move as a group. To avoid confusion we have removed the word "coherent" from this sentence (Line 221).

2.0.3 "Are there further approaches to identify coherent structures of meteorological phenomena besides the FTLE?"

We thank the Reviewer for this question. In meteorology there are many methods to identify coherent structures such as hurricanes and extratropical cyclones. However, from a purely kinematical point of view, there is a limited but growing number of approaches, such as the geodesic transport barriers of Haller and Beron-Vera (2012). 
Here the departure positions are shown in units of degrees Latitude and Longitude. We have now clarified this in the figure caption.

\subsubsection{TCWF}

Interactive

comment

We thank the reviewer for this observation. We have specified this abbreviation in the revised version.

\subsection{6 "figures are often placed far away in the paper"}

We thank the reviewer for this comment. We have rearranged the figures in the revised version.

\subsubsection{Figure 5}

We appreciate the Reviewer's comment and have added a brief explanation of the LCS computation. However, this computation is described in detail in the Methodology section.

\subsubsection{Are $x_{0}$ and $x_{1}$ in $\mathbb{R}^{2}$ ?}

Yes, $V_{\rho_{v}}$ is vertically integrated such that $V_{\rho_{v}}=V_{\rho_{v}}(x, y)$ where $x, y$ are points in $\mathbb{R}^{2}$ as they correspond to locations in the Earth's surface. Thus, the points $x_{0}$ and $x_{1}$ are in $\mathbb{R}^{2}$. We have clarified this in the revised version. 
Here we integrate trajectories for every grid point available in ERA5 grid. So there is no prior choice of trajectory. This have been clarified in the revised version.

2.0.10 "Why is the rate sigma exponential and not logarithmic"

Interactive

comment

Lyapunov exponents are typically defined exponential rates connecting the separation of trajectories at two different times. In the case of the backwards FTLE, we formulate that the separation $\delta$ at the departure time $t=t_{0}$ is equal to the the separation at the arrival time $t=t_{1}$ multiplied by an exponential term:

$$
\delta\left(t_{0}\right)=\delta\left(t_{1}\right) e^{\sigma \Delta t}
$$

The separation ratio $\delta\left(t_{0}\right) / \delta\left(t_{1}\right)$ is expressed by the largest eigenvalue of the Cauchy Green tensor. Thus, Equation 4 in the original submission can be obtained by applying the logarithm in the equation above and isolating the exponential rate $\sigma$, i.e., the FTLE.

\subsubsection{Why is $\lambda_{\max }$ at the same time an eigenvalue and a norm?}

We thank the reviewer for this comment. $\lambda_{\max }$ is not a norm and we have fixed this in the revised manuscript.

\subsubsection{Page 8: time resolution}

2 days is the length of the time window, the time resolution employed to compute the trajectories was 6 hours. We have clarified this in the revised version. 


\subsubsection{Line 160}

WCDD

We thank the reviewer for this suggestion. We have referred to Eq. 1 in the revised version.

2.0.14 How does this method handle sources and sinks

Interactive

comment

The flow $V_{\rho_{v}}$ used to calculate the trajectories should not be affected by the total amount of water in the column as the water vapour density $\rho_{v}$ is both in the numerator and denominator of Eq. 1, yielding a flow that is not dependent on the horizontal water vapour distribution. The role of $\rho_{v}$ is only to provide a vertical scaling that favours moist levels. Therefore, we hope that sources and sinks do not affect our detection of LCSs.

\subsubsection{What is the absolute number of events?}

The absolute number of events would depend on the size of the boxes and the time interval in which the events are accounted for. The fine grid we considered in this work results in the low fraction of events per box shown in Figure 6. A first order guess of the absolute number of days in which an event occurred would be something between 0 and 50 days per year, depending on the region of the domain.

\subsubsection{Motivate the correlations in 6.2}

We thank the reviewer for this comment and have included a motivating sentence as suggested. 
2.0.17 Figure 8

: We have enhanced the size of the VIMF vectors so they are clearer.

2.0.18 Lines 303-304

We have added references as requested.

\subsubsection{Lines 311-312}

We have added references as requested.
WCDD

Interactive comment 